\title{
Observations of Planetary Nebulae Haloes
}

\author{
Romano L.M. Corradi \\ Isaac Newton Group of Telescopes, Apartado de Correos 321, E-38700 \\ Sta. Cruz de la Palma, Canary Islands, Spain \\ e-mail: rcorradi@ing.iac.es
}

\begin{abstract}
.
An improved database of ionized haloes around PNe has been built by adding the results of an extensive observational campaign to the data available in the literature. The new observations allowed us to discovered new haloes around CN 1-5, IC 2165, IC 2553, NGC 2792, NGC 2867, NGC 3918, NGC 5979, NGC 6578, PB 4, and possibly IC 1747.

The global sample consists of $29 A G B$ haloes, that are believed to still contain information about the mass loss from the AGB progenitor star. Six of these haloes show a highly asymmetrical geometry that is tentatively ascribed to the interaction of the stellar outflow with the ISM.

Another $5 \mathrm{PNe}$ show candidate recombination haloes. These are produced by the recombination front that sets up when the stellar luminosity drops in its post-AGB evolution. The resulting, limb-brightened shell resembles a real AGB halo, but is not related to AGB any mass loss event.

Double AGB haloes are found in at least $4 \mathrm{PNe}$.

For $11 \mathrm{PNe}$, deep images are available, but no halo is found to a level of $\lesssim 10^{-3}$ the peak surface brightness of the inner nebula.

These observations show us that ionized haloes are a common morphological component of PNe, being found in $70 \%$ of elliptical PNe for which adequately deep images exist. Statistical properties of the haloes are briefly discussed. Using the kinematical ages of the haloes and inner nebulae, we conclude that most of the PNe with detected haloes have hydrogen burning central stars.
\end{abstract}

\section{Introduction}

Modern radiation-hydrodynamical simulations predict the existence of faint ionized haloes around the inner bright bodies of PNe (cf. D. Schönberner and M. Steffen, this conferences). They have a surface brightness some $10^{3}$ times fainter than the inner nebulae, and still contain the information about the AGB mass loss history of the progenitor stars. We therefore name them as AGB haloes.

Recovering the AGB mass loss history by studying AGB haloes is however possible only provided that detailed radiation-hydrodynamical modelling is applied, as other structures that might be confused with real AGB haloes are expected to develop in PNe under the action of winds and radiation from the evolving central stars. For instance, in evolved PNe, when the stellar luminosity 
drops in its post-AGB evolution, a recombination front sets on and the attached shell evolves toward a 'detached', limb-brightened configuration that might be confused, on a pure morphological basis, with a real AGB halo. These are called recombination haloes (Corradi et al. 2000), and clearly do not trace any AGB mass loss event.

We are carrying on a theoretical and observational project aimed at getting a better knowledge of the link between the AGB mass loss and the observations of haloes in PNe. The work is done in collaboration with Matthias Steffen, Detlef Schönberner and Mario Perinotto. Some basic observational properties of PNe haloes are presented below, while a comprehensive study, that includes an imaging atlas of PNe haloes, is presented in Corradi et al. (2002).

\section{The improved halo database}

Narrowband CCD images of Southern PNe were obtained at ESO La Silla (Chile) during several runs from 1999 to 2001 at the $3.5 \mathrm{~m}$ New Technology Telescope using EMMI or SUSI2, and on 1997 at the 1.54m Danish telescope using DFOSC. The observations of Northern PNe were carried out at La Palma (Spain) on 2000-2001 at the 1m Jacobus Kaptein Telescope using the JAG camera, at the $2.5 \mathrm{~m}$ Isaac Newton Telescope using its Wife Field Camera, and at the $2.6 \mathrm{~m}$ Nordic Optical Telescope using the ALFOSC multi-mode instrument. For each target, we obtained deep $\mathrm{H} \alpha+[\mathrm{NII}]$ and/or [OIII] images. As a general rule, we aimed to get $>10^{5}$ detector counts in the peak surface brightness of the inner nebulae, to allow for detection of extended haloes at least one thousand times fainter.

In total, we obtained images for $35 \mathrm{PNe}$. New AGB haloes were discovered in the following 10 PNe: CN 1-5, IC 2165, IC 2553, NGC 2792, NGC 2867, NGC 3918, NGC 5979, NGC 6578, PB 4, and possibly IC 1747 . New images of a number of haloes previously known from the literature were also obtained to improve or complement the information available so far. Uncertain cases, as well as non-detections, are also present.

Relevant observations about PNe haloes (mainly imaging) was also found in some 30 articles in the literature. From those, the basic properties of the inner nebulae and haloes have been extracted, such as their morphology, sizes and surface brightness. Sixty original CCD images from these works were kindly provided by the authors, and added to our observational database of PNe haloes.

By adding our observational data set with the literature data, an improved list of haloes around PNe has been compiled. It consists of:

- 20 PNe with circular or slightly elliptical 'bona fide' AGB haloes. They are: CN 1-5, IC 1454, IC 2165, IC 2553, NGC 2022, NGC 2438, NGC 2792, NGC 2867, NGC 3918, NGC 5979, NGC 6543, NGC 6720, NGC 6826, NGC 6884, NGC 6891, NGC 7009, NGC 7662, PB 4, TC 1, and VY 2-3.

- 6 PNe with highly asymmetrical AGB haloes: IC 4593, M 1-46, M 2 40, NGC 2867, NGC 6578, and NGC 6629. Interaction with the ISM (e.g. Villaver, Manchado \& García-Segura 2000) might explain the highly elongated shapes of these haloes. 
- $5 \mathrm{PNe}$ with candidate recombination haloes, namely NGC 2438, NGC 3587, NGC 6720, NGC 6853, and NGC 7293.

- 13 uncertain and peculiar cases: He 2-119, IC 4593, IC 1747, MA 3, NGC 40, NGC 3242, NGC 3699, NGC 5882, NGC 6210, NGC 6369, NGC 6751, NGC 6803, NGC 7009, and Sh 2-200.

- 11 Non-detections. They are: BD+303639, He 2-107, IC 289, IC 2003, IC 2448, IC 3568, My 60, NGC 1535, NGC 2392, NGC 6905, and NGC 7354.

\section{Halo properties}

Excluding the uncertain cases, ionized AGB haloes are found in $60 \%$ of elliptical $\mathrm{PNe}$ for which adequately deep images exist. Another $10 \%$ show recombination haloes. Thus ionized haloes should be considered as a common morphological component of PNe.

A 'universal' characteristic of all ionized haloes observed around PNe (both the elliptical, the highly asymmetrical AGB haloes and the recombination ones) is that they are limb-brightened and display substructures as knots, ridges, or azimuthal fluctuations of the surface brightness.

In the H-R diagram, PNe with AGB haloes seem to follow the standard mass distribution of the general sample of Galactic PNe, with most masses clumped around $0.6 \mathrm{M}_{\odot}$. Recombination haloes correspond to objects of low-luminosity, in the fading part of their post-AGB evolution. 'Non-detections', on the average, seem to be located at higher luminosities (CSs masses) than the sample with detected haloes.

Four to nine PNe in our sample are found to possess two AGB haloes. The second, outer halo is only few times fainter than the first one, or even of similar surface brightness as in the case of IC 2165. The size ratio between the two haloes ranges from 1.5 to 4. For two PNe, NGC 2438 and NGC 6720, we propose that one halo is a recombination halo, and the other one a genuine AGB halo (Corradi et al. 2000).

Most haloes have a radius between $0.2 \mathrm{pc}$ and $0.8 \mathrm{pc}$, as compared to typical radii for the inner nebulae of few tenths of a parsec for non-evolved $\mathrm{PNe}$, and up to almost $0.3 \mathrm{pc}$ for very evolved objects. The haloes with sizes larger than $0.8 \mathrm{pc}$ belong to $\mathrm{PNe}$ in which double haloes are found, and obviously correspond to the outermost halo. They are PB 4 (1.2 pc) and NGC $2867(\geq 1.3 \mathrm{pc})$.

From our imaging database, we have also extracted surface brightness profiles for the haloes and inner nebulae, and from them we estimated an 'average' surface brightness of the haloes edges, normalized to the peak emission in the inner nebula. In agreement with our models, most circular and slightly elliptical AGB haloes have a relative surface brightness $\leq 2.5 \times 10^{-3}$. Slightly higher brightnesses are displayed by the most evolved objects, like NGC 2438 and IC 1454. Several highly asymmetrical AGB haloes also have a surface brightness larger than the circular AGB haloes, up to nearly $10^{-2}$. Compression by the interaction with the ISM is the most likely explanation for this enhanced emission (e.g. Villaver 2001). As predicted by models (Corradi et al. 2002), recombination haloes are significantly brighter than AGB haloes, having a surface 
brightness between $0.5 \times 10^{-2}$ and $25 \times 10^{-2}$, with little overlap with genuine AGB haloes. Note that, according to the surface brightness, haloes cannot be confused with the 'attached' shells that are often observed around the inner bright rims of the PNe (e.g. Chu, Jacoby \& Arendt 1987), as these are much brighter, several tenths that of the rim peaks.

Kinematical ages for the inner nebulae, $t_{n e b}$, and for the haloes, $t_{h a l o}$, were computed assuming spherical geometry from the apparent sizes, the distances, and the expansion velocities. For the inner nebulae (rims + attached shells), we adopt the velocities found from a number of sources in the literature. $t_{n e b}$ is expected to represent the post-AGB age of the PN, i.e. the time elapsed since the central star has left the AGB (cf. Schönberner et al. 1997). As the observational information about the haloes velocities available in the literature is scarce and somewhat contradictory, to compute their kinematical ages we have adopted an expansion velocity of $10 \mathrm{~km} \mathrm{~s}^{-1}$ which, according to the models, is find to be the best 'average' value to recover $t_{\text {halo }}$. The halo age computed in this way would indicates the time elapsed since the occurrence of the last thermal pulse on the AGB and the associated mass loss increase. Having estimated $t_{n e b}$ and $t_{\text {halo }}$, we can compute $\left(t_{\text {halo }}-t_{n e b}\right)$, that is the time elapsed between the last AGB thermal pulse and the epoch at which the progenitor star has left the AGB. For circular and slightly elliptical AGB haloes, this is found to be always between 20000 and $70000 \mathrm{yr}$, with a peak around $40000 \mathrm{yr}$. Considering that most CSs have masses around $0.6 \mathrm{M}_{\odot}$, and that the interpulse timescale for such a core mass is $\sim 90000 \mathrm{yr}$ (Blöcker 1995), we conclude that most of the PNe with observed $A G B$ haloes have left the $A G B$ far from a thermal pulse, at a phase at which hydrogen burning was the dominant energy source.

Finally, 4 of the 9 PN with possible double AGB haloes have a Wolf-Rayet (WR) CS. This might suggest a peculiar mass loss history for progenitors of WR CSs, or alternatively a peculiar dynamical evolution of their mass loss outflows. However, the situation is quite complex as three PNe with WR stars are also found among 'non-detections', and other objects of this kind are included in the group of uncertain cases. The case of WR central stars deserves further observations and modelling.

\section{References}

Blöcker, T., 1995, A\&A 297, 727

Corradi, R.L.M., Schönberner, D., Steffen, M. Perinotto, M., 2000, A\&A 354, 1071

Corradi, R.L.M., Steffen, M., Schönberner, D., Perinotto, M., 2002, A\&A submitted

Chu, Y.-H., Jacoby, G.H., Arendt, R., 1987 ApJS 64, 529

Schönberner, D., Steffen, M., Stahlberg, J., Kifonidis, K., Blöcker, T., 1997, in: Advances in Stellar Evolution, Rood, R.T., Renzini, A. (eds.), Cambridge Univ. Press, 146

Villaver, E., Manchado, A., García-Segura, G., 2000, in Asymmetrical Planetary Nebulae II: From Origins to Microstructures, ASP Conference Series, Vol. 199. Edited by J. H. Kastner, N. Soker, and S. Rappaport, p. 321 